\title{
Estrutura da Crosta sob Norte do Peru usando a Tomografia Sísmica de Ruido de Ambiente
}

Cristobal Condori (1,2), George Sand França (1), Hernando Tavera (2), Colton Lynner (3), Susan Beck (3) 1 Universidade de Brasília; 2 Instituto Geofísico del Perú, 3 University of Arizona

Copyright 2018, SBGf - Sociedade Brasileira de Geofísica

Este texto foi preparado para a apresentação no VIII Simpósio Brasileiro de Geofísica, Salinópolis, 18 a 20 de setembro de 2018. Seu conteúdo foi revisado pelo Comitê Técnico do VIII SimBGf, mas não necessariamente representa a opinião da SBGf ou de seus associados. É proibida a reprodução total ou parcial deste material para propósitos comerciais sem prévia autorização da SBGf.

\section{Abstract}

We present the preliminary result of image of the upper crustal structure in northern Peru, for the propose we use cross correlation of ambient seismic noise at 26 broadband stations from national and international networks. We reconstructing Rayleigh wave Green's functions, were obtained after the frequency-domain normalization of the ambient noise recordings and stacking of 14 months of normalized data. Dispersion curves from phase velocity maps, were inverted as a function of depth to obtain 2D shear wave velocity model of the upper crust. The results reveals features that correlates with surface geology. We observed Lowvelocity zone under the Sub-Andes that may represent regions with mayor deformation caused by the isostatic compensation that correlate with the seismicity, crustal thickness model and Bouguer gravity values.

\section{Introdução}

A dinâmica e estrutura da litosfera sob a Borda Ocidental do Peru (BOP), é consequência do processo de subducção da placa litosférica de Nazca na placa continental Sul-Americana, que convergem com velocidade relativa de 57 a $59 \mathrm{~mm} / \mathrm{ano}$ (Kendrick et al., 2003). Diversos estudos foram aplicadas com a finalidade de entender a estrutura e a dinâmica da litosfera (Antonijevic et al., 2015; Bishop et al., 2017; Condori et al., 2017; Eakin et al., 2015, 2014; Eakin e Long, 2013; Kumar et al., 2016; Phillips et al., 2012). Apesar desse esforço, muitos aspectos permanecem pouco compreendidos, especialmente na região norte do Peru.

A região norte do Peru (Figura 1), apresenta heterogeneidades litológicas complexas (Tavera e Buforn, 1998), uma região com ausência de atividade vulcânica, geometria da placa de Nazca tipo subhorizontal (Gutscher et al., 2000), mudança de orientação e baixa elevação topográfica da Cordilheira dos Andes, e particularmente com uma baixa taça de recorrência de terremotos com magnitude maior a 8.0 Mw nos últimos 500 anos.

Neste estudo, aplicamos a técnica de tomografia sísmica de ruído ambiental (ANT - Ambient Noise Tomography) com o objetivo de estudar as estruturas de velocidade da crosta sob o norte do Peru.

\section{Dados e Metodologia}

Para a análise utilizamos formas de onda contínuas para 14 meses (2016 e 2017), todas registradas por 26 estacões sismográficas de banda larga correspondente às Redes Sismográficas Nacionais (Instituto Geofísico del Perú-IGP) e internacionais: a Rede Sismográfica Brasileira (RSBR) e do Equador (Figura 1).

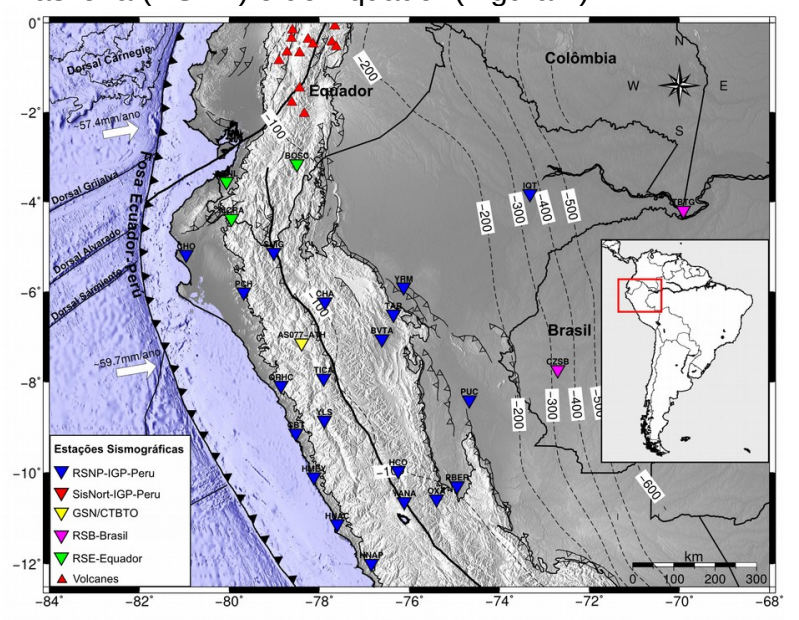

Figura 1: Principais características tectônicas e geomorfológicas dentro da área de estudo. Os triângulos invertidos indicam as estações sismográficas usadas neste estudo. As linhas tracejadas de cor pretas são os contornos da placa de Nazca do modelo Slab1.0 de Hayes et al. (2012).

A tomografia de ruido de ambiente no norte do Peru foi realizada seguindo o procedimento descrito por Bensen et al. (2008): (1) remoção da resposta do instrumento e conversão dos sismogramas para a velocidade real do solo (deslocamento), (2) dizimação para uma mostra por segundo, (3) remoção da média. Posteriormente, analisamos as funções empíricas de Green das ondas Rayleigh através da correlação cruzada (CC) do ruido sísmico ambiente entre todos os pares de estações.

A Figura 2 mostra um gráfico das funções empíricas de Green, onde mostra claramente as ondas Rayleigh em términos da distância versus tempo empilhadas para 14 meses de dados (11/2016 -12/2017).

Utilizamos a técnica de Análise de Frequência-Tempo (FTAN) (Levshin et al., 1989) para medir velocidades de fase de ondas de superfície extraídas das correlações cruzadas. O passo final foi inverter os mapas de velocidade de fase das ondas Rayleigh para obter a estrutura de profundidade, para esse propósito nos seguimos o método para tomografia de ondas de superfície de Barmin et al. (2001). O método baseia-se principalmente na minimização de uma função de regularização composta de uma função de suavização lateral em forma de Gauss e uma restrição na amplitude da perturbação ponderada pela densidade do caminho do raio local. Um exemplo desta parte do processamento apresenta-se na Figura 3. 


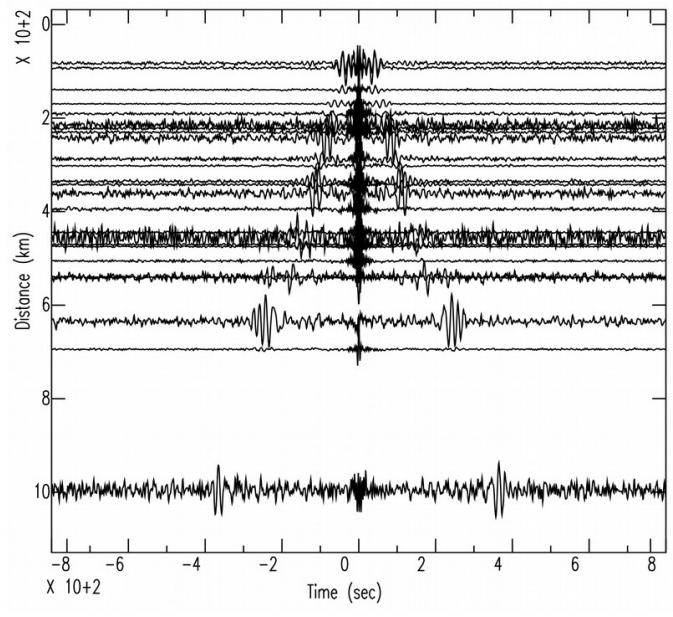

Figura 2: Registros (componentes verticais) das ondas Rayleigh obtidas das funções da correlação cruzada do ruido sísmico, empilhadas para 14 meses na estação de Atahuallpa (ATH).

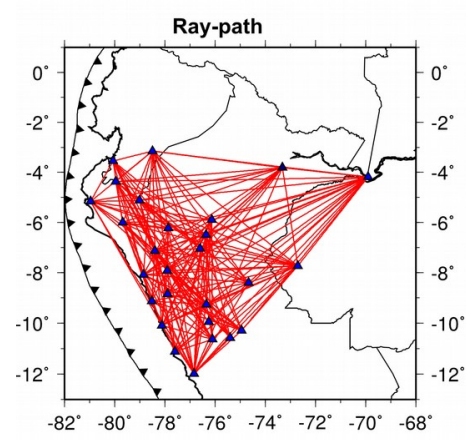

Figura 3: a) Ray-path utilizados na inversão para gerar o mapa de velocidade de fase com período igual a 20 $\mathrm{s}$.

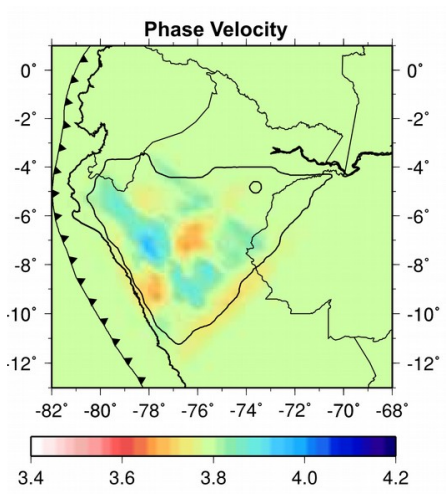

Figura 4: Mapa de velocidade de fase, mostrando os efeitos dos parâmetros de suavidades selecionadas para este estudo.

\section{Resultados}

A aplicação da técnica da extração de ondas de superfície através da correlação de ruido ambiental permitiu gerar mapas de velocidade de fase para períodos entre 10 e 25 segundos, mas neste trabalho apenas analisamos para o período $20 \mathrm{~s}$, por melhor ressaltarem as feições geológicas na área de estudo. Na Figura 5 é mostrado o mapa de velocidade da onda cisalhante (preliminar) para uma profundidade de $\sim 30$ $\mathrm{km}$. Observa-se uma anomalia de alta velocidade (3.5 - $3.9 \mathrm{~km} / \mathrm{s}$ ) sob a Cordilheira dos Andes (CA), e anomalias de baixa velocidade $(3.0-3.4 \mathrm{~km} / \mathrm{s})$ nos flancos da CA que possivelmente se trata de estruturas sujeitas a maiores esforços causados por o efeito de flexura por compensação isostática.

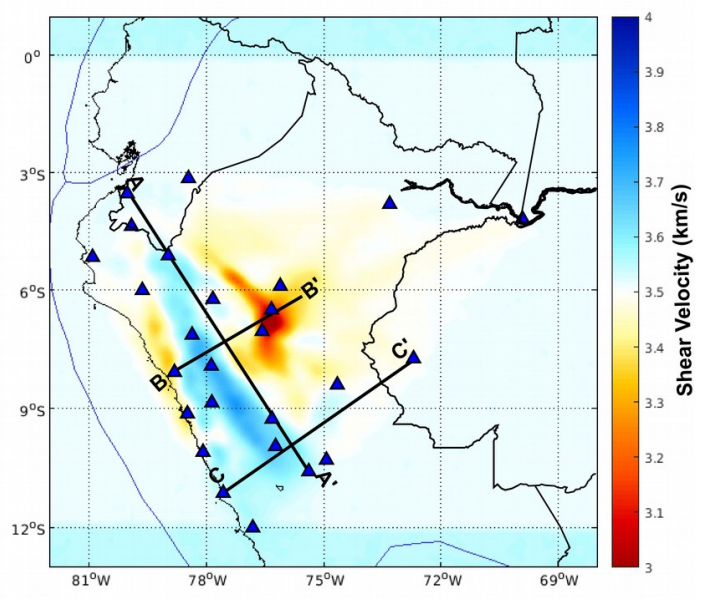

Figura 5: Mapa de velocidade da onda cisalhante obtida a partir da inversão das ondas Rayleigh para uma profundidade de $-30 \mathrm{~km}$. Os triângulos azuis indicam a localização das estacões sismográficas e as linhas de cor preta mostram a localização dos perfiles na Figura 6.

\section{Discussão e Conclusões}

Não a atividade vulcânica na região de estudo, portanto as anomalias observadas, principalmente de baixa velocidade não inferem a presença do magmatismo ou a fusão parcial.

Para um melhor análise dos resultados em profundidade, geramos três perfis cobrindo tudo a área de estudo.

O perfil A-A' com direção aproximadamente paralela à CA mostra uma possível variação lateral de estruturas com alta e baixa velocidade que coincidem com a variação topográfica em direção Norte-Sul.

No perfil B-B' observa-se uma anomalia de baixa velocidade sob a zona Sub-andina (SA), (estacões TAR, YUR e BVTA ). Nesta região a CA decresce, onde a espessura crustal $(40 \mathrm{~km})$ e a resposta gravimétrica (-35 e -75 mGal) mostram correlação.

No perfil $C-C^{\prime}$ na região central do Peru, não se evidencia anomalias com fortes contrastes de velocidade.

A anomalia de baixa velocidade observada no perfil BB' correlaciona-se com a distribuição de sismos crustais, com focos distribuídos entre 20 e $35 \mathrm{~km}$ (Figura 7). Esse fato pode estar correlacionado com fatores como diferenças composicionais entre a CA e o cráton amazônico.

\section{Agradecimentos}

Os autores agradecem a CAPES, Instituto del Geofísico del Equador, Universidade do Arizona e Instituto Geofísico del Perú. 


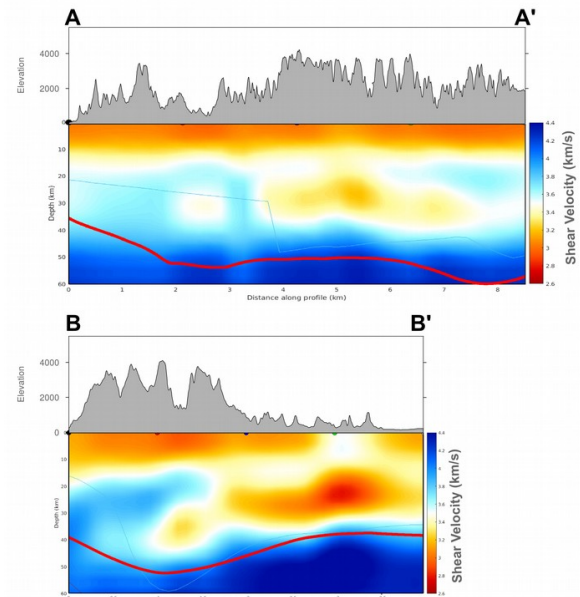

C

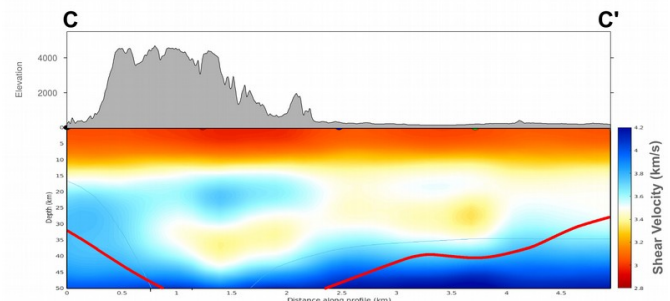

Figura 6: Secções verticais da velocidade da onda cilhante alongo das linhas (A-A', B-B' e C-C' Figura 5). linha vermelha apresenta a profundidade do Moho estimada por Condori et al. (2017).

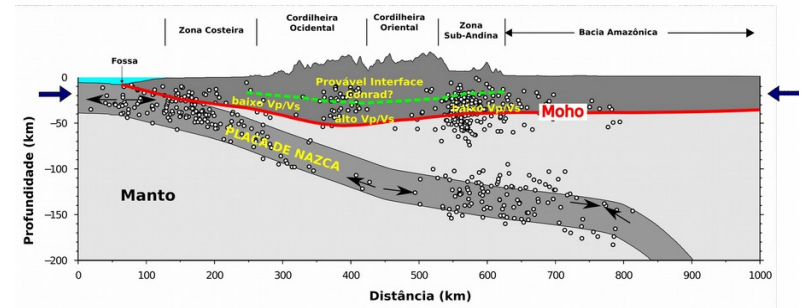

Figura 7: Modelo de subducção a partir dos resultados das estimativas de espessura crustal por Condori et al. (2017) e o modelo Slab1.0 (Hayes et al., 2012). Os pontos brancos representam os epicentros da sismicidade com magnitudes $\geq 5,0 \mathrm{Mw}$ do catálogo sísmico do IGP (1960-2016).

\section{Referências}

Antonijevic, S.K., Wagner, L.S., Kumar, A., Beck, S.L., Long, M.D., Zandt, G., Tavera, H., Condori, C., 2015. The role of ridges in the formation and longevity of flat slabs. Nature 524, 212-215. doi:10.1038/nature14648

Barmin, M.P., Ritzwoller, M.H., Levshin, A.L., 2001. A Fast and Reliable Method for Surface Wave Tomography, in: Monitoring the Comprehensive Nuclear-Test-Ban Treaty: Surface Waves. Birkhäuser Basel, Basel, p. 1351-1375. doi:10.1007/978-3-03488264-4_3

Bensen, G.D., Ritzwoller, M.H., Shapiro, N.M., 2008. Broadband ambient noise surface wave tomography across the United States. Journal of Geophysical Research 113, B05306. doi:10.1029/2007JB005248

Bishop, B., Beck, S., Zandt, G., Wagner, L., Long, M., Sanja, K., Abhash, K., Tavera, H., 2017. Causes and consequences of flat-slab subduction in southern Peru. Geosphere 13 (5), 392-1407.

Condori, C., França, G.S., Tavera, H.J., Albuquerque, D.F., Bishop, B.T., Beck, S.L., 2017. Crustal structure of north Peru from analysis of teleseismic receiver functions. Journal of South American Earth Sciences 76, 11-24. doi:10.1016/j.jsames.2017.02.006

Eakin, C.M., Long, M.D., 2013. Complex anisotropy beneath the Peruvian flat slab from frequencydependent, multiple-phase shear wave splitting analysis. Journal of Geophysical Research: Solid Earth 118, 4794-4813. doi:10.1002/jgrb.50349

Eakin, C.M., Long, M.D., Beck, S.L., Wagner, L.S., Tavera, H., Condori, C., 2014. Response of the mantle to flat slab evolution: Insights from local $S$ splitting beneath Peru. Geophysical Research Letters 41, 34383446. doi:10.1002/2014GL059943

Eakin, C.M., Long, M.D., Scire, A., Beck, S.L., Wagner, L.S., Zandt, G., Tavera, H., 2015. Internal deformation of the subducted Nazca slab inferred from seismic anisotropy. Nature Geoscience 9, 56-59. doi:10.1038/ngeo2592

Gutscher, M.A., Maury, R., Eissen, J.P., Bourdon, E., 2000. Can slab melting be caused by flat subduction? Geology 28, 535-538.

Hayes, G.P., Wald, D.J., Johnson, R.L., 2012. Slab1.0: A three-dimensional model of global subduction zone geometries. Journal of Geophysical Research 117, B01302. doi:10.1029/2011JB008524

Kendrick, E., Bevis, M., Smalley, R., Brooks, B., Vargas, R.B., Lauría, E., Fortes, L.P.S., 2003. The NazcaSouth America Euler vector and its rate of change. Journal of South American Earth Sciences 16, 125131. doi:10.1016/S0895-9811(03)00028-2

Kumar, A., Wagner, L.S., Beck, S.L., Long, M.D., Zandt, G., Young, B., Tavera, H., Minaya, E., 2016. Seismicity and state of stress in the central and southern Peruvian flat slab. Earth and Planetary Science Letters 441, 7180. doi:10.1016/j.epsl.2016.02.023

Levshin, A., Yanovskaya, T., Lander, A., Bukchin, B., Barmin, M., Ratnikova, L., Its, E., 1989. Recording, identification, and measurement of surface wave parameters, in Seismic Surface Waves in a Laterally Inhomogeneous Earth. Seismic Surface Waves in a Laterally Inhomogeneous Earth 131-182.

Norabuena, E.O., Dixon, T.H., Stein, S., Harrison, C.G.A., 1999. Decelerating Nazca-South America and Nazca-Pacific Plate motions. Geophysical Research Letters 26, 3405-3408. doi:10.1029/1999GL005394

Phillips, K., Clayton, R.W., Davis, P., Tavera, H., Guy, R., Skinner, S., Stubailo, I., Audin, L., Aguilar, V., 2012. Structure of the subduction system in southern Peru 
Estrutura da Crosta sob Norte do Peru usando a Tomografia Sísmica de Ruido de Ambiente

from seismic array data. Journal of Geophysical

Research 117, B11306. doi:10.1029/2012JB009540

Tavera, H., Buforn, E., 1998. Sismicidad y sismotectónica de Perú. Fisica de la Tierra 10, 187219. 